\title{
Two Linear Methods for Camera Calibration and Their Applications to Augmented Reality and 3D Reconstruction
}

\author{
Jang-Hwan Im, Ji-Hong Min, Hyung-Soo Ohk, and Jong-Soo Choi \\ Image Engineering Graduate School of Advanced Imaging Science \\ Multimedia, and Film Chung-Ang University \\ Dongjak-ku, Seoul 156-756, Korea \\ \{jhim, happymin,princeok,jschoi\}@imagelab.cau.ac.kr
}

\begin{abstract}
This paper presents two new compact camera calibration methods: one is derived from a calibration pattern that consists of two planes orthogonal to each other; the other is derived from a calibration pattern that consists of three planar patterns which need not to be orthogonal. In particular, these methods allow to vary the intrinsic parameters of a camera. In order to demonstrate the effectiveness of the proposed methods, two camera calibration methods are applied to an augmented reality system with a moving zoom lens camera and a $3 \mathrm{D}$ reconstruction system respectively. Two applications have shown that the proposed methods are reliable.
\end{abstract}

\section{Introduction}

Camera calibration has been an active research topic, and there are many calibration methods $[2,6,7,9]$. Recently Zhang has proposed a flexible camera calibration method [9]. The proposed method by Zhang consists of a linear equation, followed by a nonlinear refinement based on the maximum likelihood estimation [9]. If we adapt data normalization method proposed in [4], without a nonlinear iterative algorithm it can give a good calibration result. It gives a merit of time saving and a compact linear equation to calibrate a camera. Hence we shall focus on the calibration method derived from a linear equation. A shortcoming of the calibration method proposed by Zhang is that the camera internal parameters must be fixed because it needs at least three planar patterns in three images respectively. Hence zoom in and out of a camera is prohibited. This is a limitation because zoom changing is naturally to happen in many cases, for an example in a video sequence. In this paper we demonstrate that if three planar patterns in one image are observed, the shortcoming can be overcome. Furthermore, it is sufficient to calibrate a camera if two planar patterns in one image are orthogonal to each other. First a compact linear equation for camera calibration is derived using an orthogonal calibration pattern (see Fig. 1). Second the same linear equation to calibrate a camera is derived in the case that a calibration pattern which consists of three planar patterns which need not to be orthogonal (see Fig. 2). Theoretically, the linear equations proposed in this paper to calibrate a camera are the 
same as that proposed by Zhang. But if we use the proposed methods, only one image is enough to calibrate a camera, which allows to zoom in and out in sequence images. We note that in this paper radial lens distortion is ignored. Finally two camera calibration methods are applied to an augmented reality system with a moving zoom lens camera and a 3D reconstruction system respectively in order to demonstrate the effectiveness of the proposed methods.

\section{The Linear Equation for Camera Calibration Using an Orthogonal Pattern}

A camera is modeled by the usual pinhole: the relationship between a $3 \mathrm{D}$ point $\mathbf{M}=[X, Y, z, 1]^{\mathbf{T}}$ and its image projection $\mathbf{m}=[x, y, 1]^{\mathrm{T}}$ is given by

$$
\mathbf{m}=\lambda \mathbf{K}[\mathbf{R} \mid \mathbf{t}] \mathbf{M},
$$

where $\lambda$ is an arbitrary scale factor, $(\mathbf{R}, \mathbf{t}) \in \mathbf{S O} \mathbf{O}_{\mathbf{3}}(\mathbf{R}) \times \mathbf{R}^{\mathbf{3}}$, called the extrinsic parameters, and $\mathbf{K}$, called the camera intrinsic matrix, is given by

$$
\mathbf{K}=\left[\begin{array}{ccc}
f_{x} & s & o_{x} \\
& f_{y} & o_{y} \\
& & 1
\end{array}\right]
$$

with $\left(o_{x}, o_{y}\right)$ the coordinate of the principal point, $f_{x}$ and $f_{y}$ the scale factors $x$ and $y$ axes, and $s$ the parameter describing the skewness of the two image axes. $\mathbf{P}=\boldsymbol{\lambda} \mathbf{K}[\mathbf{R} \mid \mathbf{t}]$ is called the camera matrix.

\subsection{Orthogonal Constraints in the Camera Matrix}

From the camera matrix $\mathbf{P}=\lambda \mathbf{K}[\mathbf{R} \mid \mathbf{t}]$ we denote the $3 \times 3$ sub-matrix as $\mathbf{H}_{\infty}=\lambda \mathbf{K R}$. Let us denote $\mathbf{H}_{\infty}$ and $\mathbf{R}$ as $\mathbf{H}_{\infty}=\left[\mathbf{h}_{1} \mathbf{h}_{2} \mathbf{h}_{3}\right]$ and $\mathbf{R}=\left[\mathbf{r}_{1} \mathbf{r}_{2} \mathbf{r}_{3}\right]$ respectively. From $\frac{1}{\lambda} \mathbf{K}^{-1} \mathbf{H}_{\infty}=\mathbf{R}$ we have

$$
\frac{1}{\lambda} K^{-1} h_{1}=r_{1}, \frac{1}{\lambda} K^{-1} h_{2}=r_{2}, \frac{1}{\lambda} K^{-1} h_{3}=r_{3} .
$$

Since $\mathbf{R}^{\mathrm{T}} \mathbf{R}=\mathbf{I}$, that is, $\left\|\mathbf{r}_{\mathbf{i}}\right\|=\mathbf{1}, \mathrm{i}=1,2,3$ and $\mathbf{r}_{\mathbf{i}}^{\mathrm{T}} \mathbf{r}_{\mathbf{j}}=\mathbf{0}$ for $i \neq j$, we have

$$
\begin{aligned}
& \mathbf{h}_{1}^{\mathrm{T}} \mathbf{K}^{-\mathrm{T}} \mathbf{K}^{-1} \mathbf{h}_{2}=\mathbf{h}_{2}^{\mathrm{T}} \mathbf{K}^{-\mathrm{T}} \mathbf{K}^{-1} \mathbf{h}_{3}=\mathbf{h}_{3}^{\mathrm{T}} \mathbf{K}^{-\mathrm{T}} \mathbf{K}^{-1} \mathbf{h}_{1}=\mathbf{0}, \\
& \mathbf{h}_{1}^{\mathrm{T}} \mathbf{K}^{-\mathrm{T}} \mathbf{K}^{-1} \mathbf{h}_{1}=\mathbf{h}_{2}^{\mathrm{T}} \mathbf{K}^{-\mathrm{T}} \mathbf{K}^{-1} \mathbf{h}_{2}=\mathbf{h}_{3}^{\mathrm{T}} \mathbf{K}^{-\mathrm{T}} \mathbf{K}^{-1} \mathbf{h}_{3} .
\end{aligned}
$$


The matrix $\mathbf{K}^{-\mathbf{T}} \mathbf{K}^{-1}$ can be considered as the conic coefficient matrix that has five degree of freedoms. A conic is determined uniquely (up to scale) by five points in general position [4]. From (1) and (2) we have six constraints being able to determine $\mathbf{K}^{-\mathbf{T}} \mathbf{K}^{-\mathbf{1}}$. Then $\mathbf{K}$ is obtained from $\mathbf{K}^{-\mathbf{T}} \mathbf{K}^{-\mathbf{1}}[3,9]$. The extrinsic parameters $\mathbf{R}, \mathbf{t}$, are calculated from the relation $\mathbf{P}=\lambda \mathbf{K}[\mathbf{R} \mid \mathbf{t}]$.

\section{Algorithm: Camera Calibration Method 1}

1. Determine the projection matrix $\mathbf{P}=\lambda \mathbf{K}[\mathbf{R} \mid \mathbf{t}]$, which is proposed in [4, Chap. 6].

2. Compute the camera intrinsic matrix $\mathbf{K}$ using the method proposed by this paper.

3. Compute $\mathbf{R}, \mathbf{t}$ from the computed $\mathbf{K}$ and the relation $\mathbf{P}=\lambda \mathbf{K}[\mathbf{R} \mid \mathbf{t}]$.

We note that the computed rotation matrix $\mathbf{R}$ can not satisfy the properties of a rotation matrix. Given $\mathbf{R}$ there is a method to estimate the best rotation matrix [3].

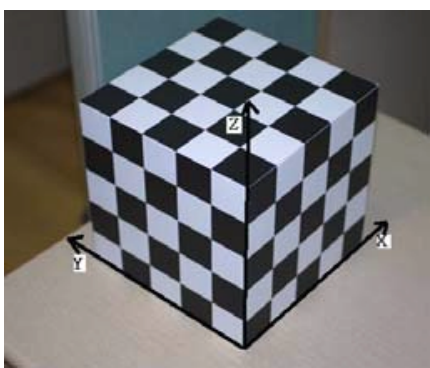

Fig 1. In this calibration pattern we need only two orthogonal planes, for an example $<\mathrm{x}, \mathrm{z}>$ and $<\mathrm{y}, \mathrm{z}>$ planes.

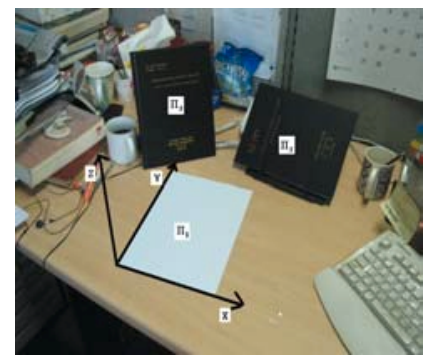

Fig 2. Three planar patterns in an image are sufficient to calibrate a camera.

\section{The Linear Equation for Camera Calibration Using Three Planar Patterns that need not to be Orthogonal}

We have the freedom to choose the world coordinate frame [4]. As shown Fig 2, we choose a world coordinate system such that the plane $\Pi_{1}$ is the XY-plane, that is, the plane $\Pi_{1}$ have zero Z-coordinate. Then the image of a point on $\Pi_{1}$ is given by

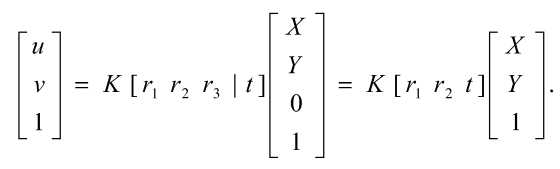

Hence we get a homography between the plane $\Pi_{1}$ and the corresponding image:

$$
\mathbf{H}_{1}=\lambda \mathbf{K}\left[\mathbf{r}_{1} \mathbf{r}_{2} \mathbf{t}\right] \text {. }
$$


Let $\mathbf{H}_{1}\left[\mathbf{h}_{11} \mathbf{h}_{12} \mathbf{h}_{13}\right]$. Since $\mathbf{r}_{1}^{\mathrm{T}} \mathbf{r}_{2} \quad \mathbf{0}$ and $\left\|\mathbf{r}_{1}\right\|\left\|\mathbf{r}_{\mathbf{2}}\right\| \mathbf{1}$, we get the following two equations:

$$
\mathbf{h}_{11}^{\mathrm{T}} \mathbf{K}{ }^{\mathrm{T}} \mathbf{K}{ }^{1} \mathbf{h}_{12} \quad \mathbf{0}, \quad \mathbf{h}_{11}^{\mathrm{T}} \mathbf{K}{ }^{\mathrm{T}} \mathbf{K}{ }^{1} \mathbf{h}_{11} \quad \mathbf{h}_{12}^{\mathrm{T}} \mathbf{K}{ }^{\mathrm{T}} \mathbf{K}{ }^{1} \mathbf{h}_{12} .
$$

In the same way we can get homographies between the planes $3_{1}, 3_{2}$ and the corresponding images respectively: $\mathbf{H}_{2} \quad\left[\begin{array}{lllll}\mathbf{h}_{21} & \mathbf{h}_{22} \mathbf{h}_{23}\end{array}\right], \mathbf{H}_{3}\left[\begin{array}{ll}\mathbf{h}_{31} \mathbf{h}_{32} & \mathbf{h}_{33}\end{array}\right]$. We have the following four equations:

$$
\begin{aligned}
& \mathbf{h}_{21}^{\mathrm{T}} K^{\mathrm{T}} \mathrm{K}^{1} \mathbf{h}_{22} \quad \mathbf{0 ,} \mathbf{h}_{21}^{\mathrm{T}} \mathrm{K}^{\mathrm{T}} \mathrm{K}^{1}{ }^{1} \mathbf{h}_{21} \quad \mathbf{h}_{22}^{\mathrm{T}} K^{\mathrm{T}} K^{1} h_{22}, \\
& h_{31}^{\mathrm{T}} K{ }^{\mathrm{T}} K{ }^{1} h_{32} \quad 0, h_{31}^{\mathrm{T}} K{ }^{\mathrm{T}} K{ }^{1} h_{31} \quad h_{32}^{\mathrm{T}} K{ }^{\mathrm{T}} K{ }^{1} h_{32} \text {. }
\end{aligned}
$$

Hence the matrix $\mathbf{K}$ is obtained from the equations (4), (5), and (6) using the method mentioned in section 2 .

\section{Algorithm: Camera Calibration Method 2}

1. Determine the homographies $\mathbf{H}_{1}, \mathbf{H}_{2}, \mathbf{H}_{3}$, which is proposed in [4, Chap. 3].

2. Compute the camera intrinsic matrix $\mathbf{K}$ using the equation (4), (5), and (6).

\section{Application of Camera Calibration Method 1 to an Augmented Reality System}

There has been much research for augmented reality based on computer vision $[1,5$, 8]. These works assume that the internal parameters are fixed and try to compute the pose of the camera. But zoom changing is naturally to happen in a video sequence. The calibration method 1 gives a solution to overcome such a limitation, and is applied to an augmented reality system. A sequence of images containing a box is obtained from a moving zoom lens camera as shown in Fig. 3. This box instead of an orthogonal calibration pattern can be used to calibrate a camera in each image using the calibration method 1 . In order to extract a control object, the chroma-key method is used. After the chroma-key process each image is converted to gray scale one. The edges of object region (the box) is extracted using the Sobel edge detector.
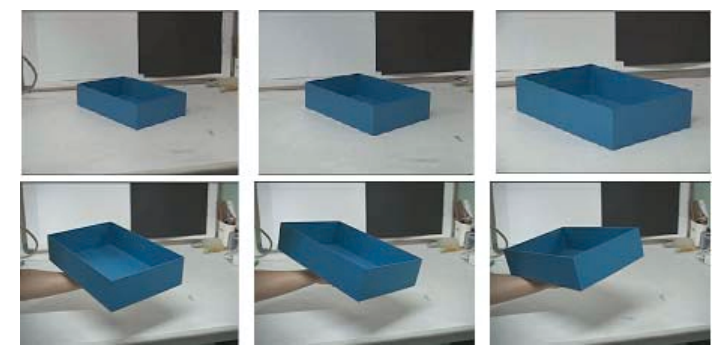

Fig 3: A sequence of images was taken by a moving zoom lens camera 
Each optimized line is obtained from the points of each edge using the least square method. The corner points of the box are obtained from the intersection of the optimized lines. The camera calibration is performed by the calibration method 1. Finally the virtual object is overlaid with each real image through the occlusion processing. The overall procedure is shown in Fig. 5.
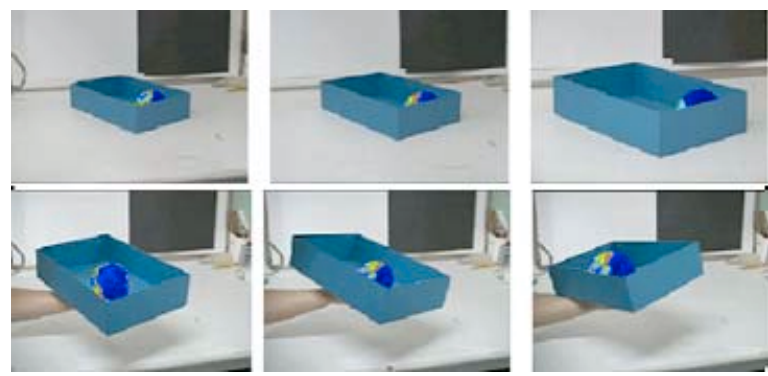

Fig 4: The earth, the computer generated image, inserted in each image, so that the generated images appear to be augmented like a part of the original scenes

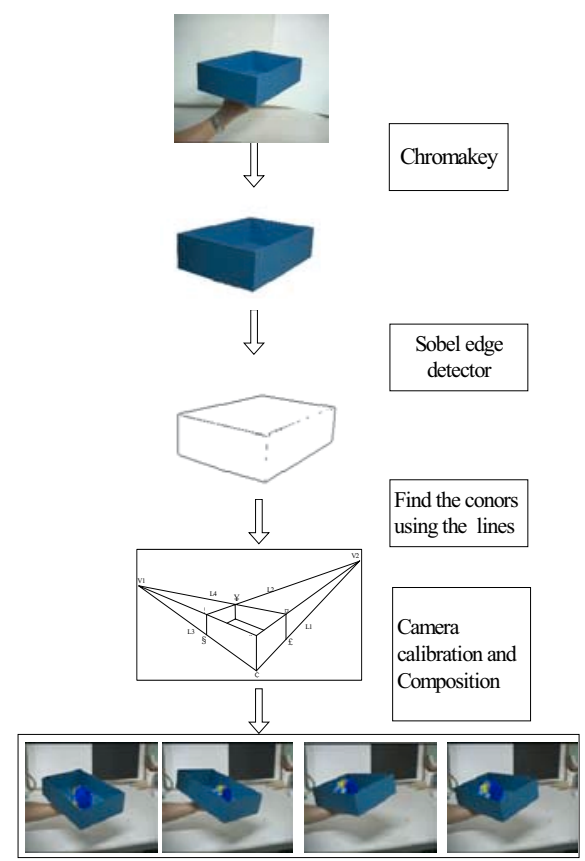

Fig 5: Overall procedure of the augmented reality system

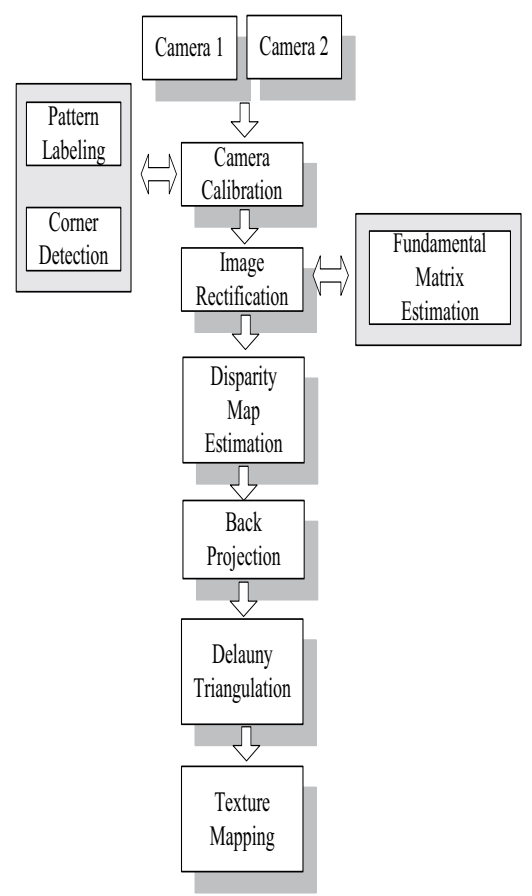

Fig 7: Overall procedure of the $3 \mathrm{D}$ reconstruction system using the proposed calibration method 2

Fig. 4 shows that the earth, the virtual object, is overlaid with real images. The rolling motion of the earth in the box is computed from the orientation of the top plane. 


\section{Application of Camera Calibration Method 2 to a 3D Reconstruction System}

Two images of a bear puppet containing three planar patterns are taken by the same camera as shown in Fig 6. Each image is calibrated by the calibration method 2 using the three planar patterns.
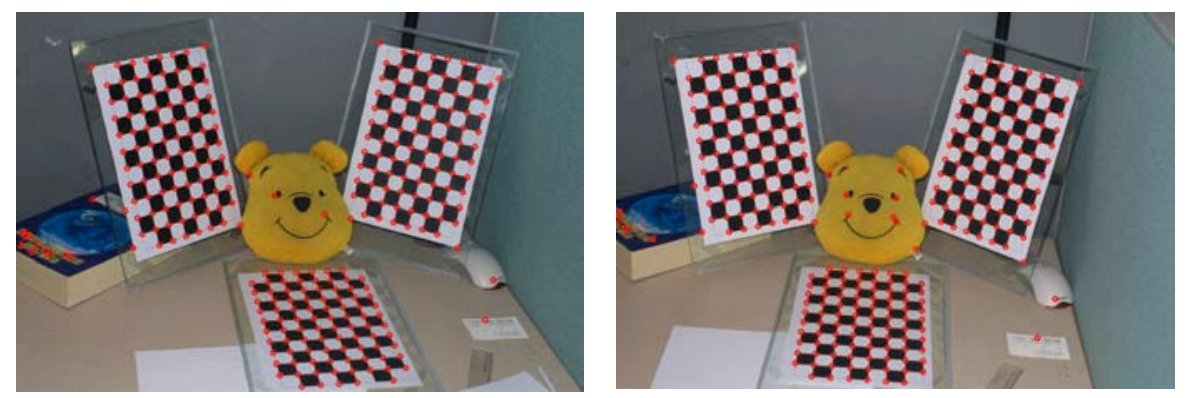

Fig 6: Two images of a bear puppet containing three planar patterns

Fig. 7 shows the overall procedure of the 3D reconstruction system proposed in this paper. Four rendered views are shown in Fig. 8.
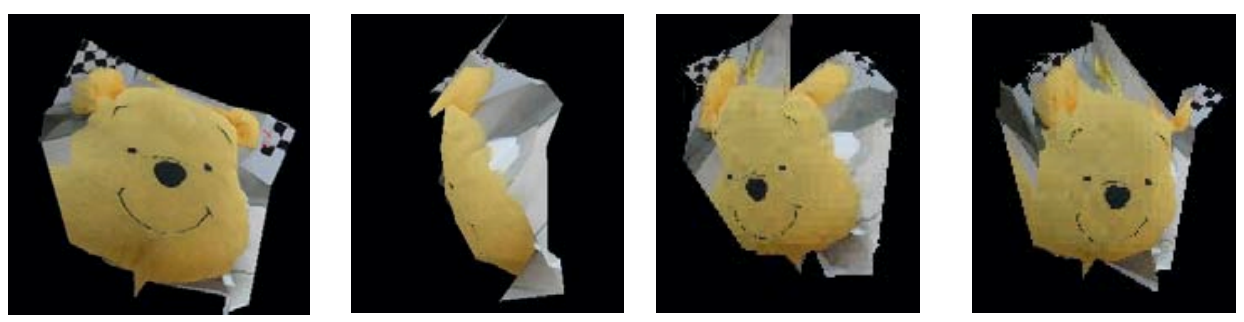

Fig 8: Four rendered views of the reconstructed the bear puppet

\section{Conclusion}

In this paper we have proposed two new compact calibration methods. We have applied two algorithms to the augmented reality system with a moving zoom lens camera and the $3 \mathrm{D}$ reconstruction system respectively, so as to test stability of the proposed algorithms. Two applications have shown that the proposed methods are reliable. 


\section{Acknowledgments}

This research was supported in part by the Ministry of Education, Seoul, Korea, under the BK21 Project, and by the Ministry of Science and Technology, Seoul, Korea, under the NRL Project (2000-N-NL-01-C-285).

\section{References}

[1] R. T. Azuma and G. Bishop, "Improving static and dynamic registration in an optical see through display", In Proc. SIGGRAPH'94, pages 194-204.

[2] B. Caprile and V. Torre, "Using Vanishing Points for Camera Calibration," The International Journal of Computer Vision, 4(2): 127-140, 1990.

[3] G. Golub and C. van Loan, Matrix Computations, The John Hopkins University, Baltimore, Maryland, 3 edition, 1996.

[4] R. Hartely and A. Zissermann, Multiple View Geometry in Computer Vision, Cambridge University Press, 2000.

[5] G. Simon and M. -O. Berger, "A Two-stage Robust Statistical Method for Temporal Registration from Features of Various Type”, In Proc. ICCV'98, pages 261-266.

[6] B. Triggs, "Auto-calibration and the absolute quadric", In Proc. IEEE Conference on Computer Vision and Pattern Recognition, pages 609-614, 1997.

[7] R. Y. Tsai, "A versatile camera calibration technique for high-accuracy 3D machine vision metrology using off-the-shelf tv cameras and lenses", IEEE Journal of Robotics and Automation, 3(4):323-344, 1987.

[8] M. Uenohara and T. Kanade, "Vision based object registration for real time image overlay", Journal of Computers in Biology and Medecine, 1996.

[9] Z. Zhang, "A flexible new technique for camera calibration", IEEE Transactions on Pattern Analysis and Machine Intelligence, 22(11):1330-1334, 2000. 